\title{
Evaluating Effect of Cognitive Behavioral Nursing on Quality of Life in Hemodialysis Patients
}

\author{
Huixia Yu ${ }^{1}$, Yu Meng ${ }^{1}, J_{i a n} \mathrm{Li}^{1}$, Sijing Shao ${ }^{1}$, Jinglan $\mathrm{Luo}^{2}$, Xiutin $\mathrm{Li}^{3}$, * \\ ${ }^{1}$ Blood Purifying Center, The First Affiliated Hospital, Jinan University, Guangzhou, China \\ ${ }^{2}$ Internal Medicine Department, The First Affiliated Hospital, Jinan University, Guangzhou, China \\ ${ }^{3}$ Department of Gastroenterology and Rheumatology, The First Affiliated Hospital, Jinan University, Guangzhou, China \\ Email address: \\ 672848038@qq.com (Huixia Yu), elajob@126.com (Yu Meng), 137905065@qq.com (Jian Li), 395133258@qq.com (Sijing Shao), \\ tlj1@jnu.edu.cn (Jinglan Luo),503549284@qq.com (Xiutin Li) \\ ${ }^{*}$ Corresponding author
}

\section{To cite this article:}

Huixia Yu, Yu Meng, Jian Li, Sijing Shao, Jinglan Luo, Xiutin Li. Evaluating Effect of Cognitive Behavioral Nursing on Quality of Life in Hemodialysis Patients. American Journal of Internal Medicine. Vol. 8, No. 5, 2020, pp. 246-249. doi: 10.11648/j.ajim.20200805.19

Received: September 13, 2020; Accepted: September 22, 2020; Published: September 30, 2020

\begin{abstract}
Objective: To assess effect of cognitive behavioral nursing on quality of life in hemodialysis patients. Methods: We invite 120 patients to join our study from January 2018 to January 2020. They were diagnosed as chronic renal failure in the hospital. In addition, the patients receive hemodialysis treatment in treatment process. In study beginning step, the participants were randomly assigned to a control group $(n=60)$ and intervention group $(n=60)$. For participants of control group, the patients receive the common nursing intervention in hemodialysis process. Additionally, the intervention group patients receive cognitive behavioral nursing on the basis of routine nursing. Result: In depression assessment and anxiety assessment, the control group and intervention group have similar assessment in depression status and anxiety status. In after nursing intervention part, the two groups have different assessment in research result. In health literacy assessment, the two group patients improve their health literacy in four elements. In addition, intervention group has better improvement that that of control group. In addition, the research result of coping style assessment and quality of life assessment are similar, that the two groups have different improvement in research result, that intervention group has better improvement than that of control group. Conclusion: the cognitive behavioral nursing improves some aspects of chronic renal failure patients, that include mental health, health literacy of patient, coping style level of patient and quality of life.
\end{abstract}

Keywords: Hemodialysis, Chronic Renal Failure, Nursing

\section{Introduction}

Chronic renal failure is a common disease in the department of nephrology, that its etiology mainly comes from the chronic kidney function injury caused by many kinds of kidney diseases [1]. In chronic kidney function injury process, endotoxin in the body cannot be metabolized in time, and toxin retention in the body of patients will cause damage to their kidney function, forming a vicious circle as patient's chronic kidney function was injured [2,3]. Base on the reports, hemodialysis can effectively remove the toxin molecules in the blood and control the progression of the patient's disease in chronic kidney patients $[4,5]$. However, in hemodialysis process, patients affected by the condition, patient easy to produce bad emotion. As patient's poor health behavior, it is not conducive to their disease control [6].

In Krespi's research report, it indicated the lifestyle of patients on hemodialysis is strict as patient's requirements often cause significant emotional, physical, and mental stress, resulting in a loss of autonomy, independence, and function [7, 8]. In addition, hemodialysis patients are exposed to non-compliance risks in a wide range of self-care management areas as the hemodialysis patients lack cognitive behavioral nursing in treatment process. The patients lack cognition of kidney failure and personal safety experience of kidney failure $[9,10]$. The aim of this study is evaluating the effect of cognitive behavioral nursing on quality of life in hemodialysis patients. 


\section{Methods}

\subsection{Participants Enrollment and Survey Methods}

We invite 120 patients to join our study from January 2018 to January 2020. They were diagnosed as chronic renal failure in the hospital. In addition, the patients receive hemodialysis treatment in treatment process. In study beginning step, the participants were randomly assigned to a control group $(n=60)$ and intervention group $(\mathrm{n}=60)$. For participants of control group, the patients receive the common nursing intervention in hemodialysis process. Additionally, the intervention group patients receive cognitive behavioral nursing on the basis of routine nursing. About collected data, it includes depression assessment and anxiety assessment, health literacy assessment, coping style assessment, quality of life assessment. The questionnaire includes the following: Self-Rating Anxiety Scale (SAS), Self-rating depression scale (SDS), Health Literacy Surveillance Rapid Assessment Questionnaire, (HLSRAQ), Simple coping Style questionnaire, World Health Organization Quality of Life Instrument (WHOQOL-BREF) [11-15].

In detail of cognitive behavioral nursing, it contains: (1) Cognitive nursing: with the help of health knowledge manuals and videos, the patient is given detailed information about chronic renal failure, and then the patient is given information about hemodialysis. In addition, we communicate with patients to evaluate whether their irrational beliefs have objective basis. We will remove non-patient rational beliefs and restore rational beliefs. (2) Behavioral nursing: We let patients understand the importance of diet control. In this process, we listed the dietary precautions, smoking cessation and alcohol, avoiding greasy, spicy and pungent food, and explained to patients how to calculate the amount of fluid in and out. In addition, we collected and recorded the amount of fluid in and out of patients, and controlled the daily water intake and rest schedule. We will record the patient's bedtime, sleep time, wake time and wake time. At the same time, we adjust patients' sleep and rest habits to help them develop a better healthy rest and rest. In the relationship between the hospital and the patient, we strengthen the contact with the patient's family, adjust the patient's behavior, and require the patient's family to do a good job of monitoring the patient's health behavior.

\subsection{Statistical Analysis}

Our data analyzer performed the statistical analysis by SPSS 22.0. The $\mathrm{P}$ value, t-test and chi-square test were associated with collection result were analyzed. Besides, the mean standard deviation for statistical description.

\section{Result}

In table 1 , it indicates depression assessment and anxiety assessment of two groups, that the higher score means the patients had more serious depression or more serious anxiety. In before nursing intervention part, the control group and intervention group have similar assessment in depression status and anxiety status (54.39 \pm 6.86 vs $55.47 \pm 6.53$, $54.21 \pm 6.90$ vs $55.28 \pm 6.61$ ). In after nursing intervention part, the two groups have different assessment in research result. The intervention group patients have better performance in SAS research and SDS research than that of control group patients.

Table 1. Depression Assessment and Anxiety Assessment by SAS and SDS (Mean $\pm S D)$.

\begin{tabular}{llll}
\hline Projects & Period & SAS & SDS \\
\hline \multirow{2}{*}{ Control group $(\mathrm{n}=60)$} & $\mathrm{BN}$ & $54.39 \pm 6.86$ & $55.47 \pm 6.53$ \\
& $\mathrm{AN}$ & $47.10 \pm 5.27$ & $48.59 \pm 5.42$ \\
Intervention group $(\mathrm{n}=60)$ & $\mathrm{BN}$ & $54.21 \pm 6.90$ & $55.28 \pm 6.61$ \\
& $\mathrm{AN}$ & $44.74 \pm 4.93$ & $45.16 \pm 5.07$ \\
\hline
\end{tabular}

$\mathrm{BN}=$ Before nursing intervention. $\mathrm{AN}=$ After nursing intervention.

The Table 2 shows the health literacy assessment of patient, it contains health knowledge, health beliefs, health behavior and health skills. In before nursing intervention part, the patients of two group have best performance in health knowledge assessment ( $5.04 \pm 1.26$ vs $5.17 \pm 1.32)$, and the two groups have similar assessment in result. In after nursing intervention part, the two group patients improve their health literacy in four elements. In addition, intervention group has better improvement that that of control group.

Table 2. Health literacy assessment (Mean $\pm S D)$.

\begin{tabular}{|c|c|c|c|c|c|}
\hline Projects & Period & Health knowledge & health beliefs & health behavior & health skills \\
\hline \multirow{2}{*}{ Control group $(n=60)$} & $\mathrm{BN}$ & $5.04 \pm 1.26$ & $4.59 \pm 1.02$ & $4.82 \pm 1.21$ & $4.35 \pm 1.14$ \\
\hline & $\mathrm{AN}$ & $7.21 \pm 1.55$ & $6.54 \pm 1.35$ & $6.85 \pm 1.43$ & $6.39 \pm 1.50$ \\
\hline \multirow{2}{*}{ Intervention group $(n=60)$} & $\mathrm{BN}$ & $5.17 \pm 1.32$ & $4.67 \pm 1.14$ & $4.89 \pm 1.25$ & $4.47 \pm 1.16$ \\
\hline & $\mathrm{AN}$ & $8.35 \pm 1.59$ & $7.73 \pm 1.56$ & $8.42 \pm 1.47$ & $7.72 \pm 1.63$ \\
\hline
\end{tabular}

$\mathrm{BN}=$ Before nursing intervention.

$\mathrm{AN}=\mathrm{After}$ nursing intervention.

In patients' coping style assessment, the patient's reorganization of self-cognition and seek social support are about 73 score in two group research $(72.53 \pm 6.12$ vs $72.67 \pm 6.15,73.27 \pm 6.45$ vs $73.40 \pm 6.39$ ). Additionally, their escape fantasy assessment has similar score in result
$(60.38 \pm 8.47$ vs $60.29 \pm 8.51)$. In after nursing intervention part, the two groups have different improvement in research result, that intervention group has better improvement than that of control group (Table 3). 
Table 3. Coping style assessment (Mean $\pm S D)$.

\begin{tabular}{lllll}
\hline Projects & Period & Reorganization of self-cognition & Seek social support & Escape fantasy \\
\hline \multirow{2}{*}{ Control group $(\mathrm{n}=60)$} & $\mathrm{BN}$ & $72.53 \pm 6.12$ & $73.27 \pm 6.45$ & $60.38 \pm 8.47$ \\
& AN & $80.09 \pm 7.24$ & $81.14 \pm 7.59$ & $51.62 \pm 6.93$ \\
\multirow{2}{*}{ Intervention group $(\mathrm{n}=60)$} & $\mathrm{BN}$ & $72.67 \pm 6.15$ & $73.40 \pm 6.39$ & $60.29 \pm 8.51$ \\
& AN & $86.95 \pm 7.69$ & $86.33 \pm 8.10$ & $46.27 \pm 6.12$ \\
\hline
\end{tabular}

$\mathrm{BN}=$ Before nursing intervention.

$\mathrm{AN}=\mathrm{After}$ nursing intervention.

In quality of life research, it indicates patient's quality of life before nursing intervention and after nursing intervention (Table 4). In before nursing intervention group, the two groups have similar quality of life, that physiological assessment and environment assessment are approximately 69 and psychological assessment and social relations assessment are approximately 70 . On the other hand, intervention group patients have better performance in those 4 parts than that of control group.

Table 4. Quality of life assessment (Mean $\pm S D)$.

\begin{tabular}{|c|c|c|c|c|c|}
\hline Projects & Period & The physiological & psychological & The environment & Social relations \\
\hline \multirow{2}{*}{ Control group $(n=60)$} & $\mathrm{BN}$ & $69.56 \pm 5.09$ & $70.38 \pm 5.20$ & $69.27 \pm 4.81$ & $70.09 \pm 5.18$ \\
\hline & $\mathrm{AN}$ & $77.09 \pm 6.53$ & $78.12 \pm 6.17$ & $76.35 \pm 5.03$ & $77.94 \pm 5.23$ \\
\hline \multirow{2}{*}{ Intervention group $(n=60)$} & $\mathrm{BN}$ & $69.68 \pm 5.04$ & $70.52 \pm 5.13$ & $69.38 \pm 4.75$ & $70.20 \pm 5.04$ \\
\hline & AN & $82.45 \pm 6.37$ & $83.39 \pm 6.28$ & $81.26 \pm 5.14$ & $82.57 \pm 5.69$ \\
\hline
\end{tabular}

$\mathrm{BN}=$ Before nursing intervention.

$\mathrm{AN}=\mathrm{After}$ nursing intervention.

\section{Discussion and Conclusion}

Chronic renal failure from most kidney diseases in end stage. In process of chronic renal failure, patient's glomerular filtration rate will be declined, it causes a rise in blood uremic nitrogen so that patients are accompanied by cardiovascular and other diseases $[16,17]$. Chronic renal failure significantly reduces the lifespan of patients. Chronic renal failure patients have an increased risk of cardiovascular disease and may develop end stage renal disease [18]. In fact, hemodialysis is the main clinical method to treat chronic renal failure. It mainly extracorporeal circulation device to introduce patients' blood into the dialyzer, and activated carbon and adsorbent to adsorb and filter the toxin molecules in the blood [19].

Base on the results above, the cognitive behavioral nursing improves some aspects of chronic renal failure patients, that include mental health, health literacy of patient, coping style level of patient and quality of life. In depression assessment and anxiety assessment, the intervention group patients have better mental health than that of control group patients, that is similar to Aishath's report, the mental health of the patient have small improvement [20]. In health literacy research and coping style research, they are similar, that they have moderate improvement in the intervention group report result. The result is similar to Gema's research, but intervention group patients have better performance in Gema's research [21]. The quality of life research indicates the quality of life of two groups patients are improved after nursing intervention, but cognitive behavioral nursing provides more effect in the outcome.

\section{References}

[1] Chin, Andrew I. Nguyen, Tuan A. Dinesh, Kumar P. et al. Late acceleration of glomerular filtration rate decline is a risk for hemodialysis catheter use in patients with established nephrology chronic kidney disease care. Hemodialysis international. 2015; 19 (3): 379-385.

[2] Xavier, Vivian Bertoni, Roxo, Renata Sposito, Miorin, Luiz Antonio et al. Impact of continuous positive airway pressure (CPAP) on the respiratory capacity of chronic kidney disease patients under hemodialysis treatment. International urology and nephrology. 2015; 47 (6): 1011-1016.

[3] Bansal, Nisha, Mc Culloch, Charles E. Rahman, Mahboob et al. Blood Pressure and Risk of All-Cause Mortality in Advanced Chronic Kidney Disease and Hemodialysis The Chronic Renal Insufficiency Cohort Study. Hypertension. 2015; 65 (1): 93-100.

[4] Afeng M. Effects of cognitive behavioral nursing on quality of life and quality of sleep in hemodialysis patients in Department of Nephrology. Nursing Practice and Research. 2019; 16 (15): 73-74.

[5] Ozdemir, Ozturk, Kayatas, Mansur, Cetinkaya, Selma et al. Bcii-RFLP profiles for serum amiloid A1 and mutated MEFV gene prevalence in chronic renal failure patients requiring long-term hemodialysis. Renal Failure. 2015; 37 (2): 292-296.

[6] NationalChronicKidneyDiseaseFactSheet. US Department of Health and Human Services, Centers for Disease Control and Prevention; 2017 Accessed 26 February 2020.

[7] Krespi MR, Bone M, Ahmad R, Worthington B, Salmon P. Hemodialysis patients' evaluation of their lives. TurkPsikiyatri Derg. 2018; 19 (4): 365-372. 
[8] Guerra-Guerrerro V, Plazas Mdel P, Cameron BL, Salas AV, Cofre González CG. Understanding the life experience of people on hemodialysis: adherence to treatment and quality of life. Nephrol NursJ. 2016; 41 (3): 289-298.

[9] Obialo CI, Hunt WC, Bashir K, Zager PG. Relationship of missed and shortened hemodialysis treatments to hospitalization and mortality: observations from a US dialysis network. Clin Kidney J. 2017; 5 (4): 315-319.

[10] Qudah B, Albsoul-Younes A, Alawa E, Mehyar N. Role of clinical pharmacist in the management of blood pressure in dialysis patients. Int J Clin Pharm. 2016; 38 (4): 931-940.

[11] White D, Leach C, Sims R, Atkinson M, Cottrell D. Validation of the Hospital Anxiety and Depression Scale for use with adolescents. Br J Psychiatry 1999; 175: 452-454.

[12] El-Rufaie O, Absood G. Validity study of the Hospital Anxiety and Depression Scale among a group of Saudi patients. Br J Psychiatry 1987; 151: 687-688.

[13] WHOQOL-Group. The world health organization quality of life assessment (WHOQOL): position paper from the world health organization. SocSciMed 1995; 41: 1403-9.

[14] Du XB, HAN TG, Jing CX, Zhuang RS. Construction and Validation of the National HLSRAQ. Health education and health promotion, 2019, 14 (04): 310-313.
[15] Kevorkian. A preliminary study on the reliability and validity of the Simple coping style scale. Chinese Journal of Clinical Psychology. 1998; 6 (2): 114-115.

[16] Lo J, Go AS, Chertow GM, McCulloch CE, Fan D, Ordonez JD, Hsu CY. Dialysis-requiring acute renal failure increases the risk of progressive chronic kidney disease, Kidney Int. 2019; 76 (8): 893-899.

[17] Wang F, Zhang BH, Lu XQ, Wang P, Efficacy of different hemodialysis methods on dendritic cell marker CD40 and CD80 and platelet activation markerCD62P and P10 in patients with chronic renal failure, J. Clin. Lab. Anal. 2019; 33 (3).

[18] Bruck K, et al. CKD pre-valence varies across the european general population, J. Am. Soc. Nephrol. 2016; 27 (7): 2135 2147.

[19] Aktas, Serdar, Sagdik, Haci Murat, Aktas, Hatice et al. Tear function in patients with chronic renal failure undergoing hemodialysis. Renal Failure. 2015; 37 (2): 245-248.

[20] Aishath AA, Rajan G, Zeena MF, et al. Gastric trichobezoar in an end-stage renal failure and mental health disorder presented with chronic epigastric pain: A case report. Annals of Medicine and Surgery. 2020; 58: 76-79.

[21] Gema CR, Francesc M, et al. Health literacy and chronic kidney disease. Nefrología. 2017; 37 (2): 115-117. 